\title{
Muon bundle reconstruction with KM3NeT/ORCA using graph convolutional networks
}

\author{
Stefan Reck*, Thomas Eberl and Uli Katz on behalf of the KM3NeT Collaboration \\ (a complete list of authors can be found at the end of the proceedings)
}

Universität Erlangen-Nürnberg, Erwin-Rommel-Str. 1, 91058 Erlangen, Germany

Erlangen Centre for Astroparticle Physics (ECAP)

E-mail: stefan.reck@fau.de, thomas.eberl@fau.de, uli.katz@fau.de

KM3NeT/ORCA is a water-Cherenkov neutrino detector, currently under construction in the Mediterranean Sea at a depth of 2450 meters. The project's main goal is the determination of the neutrino mass hierarchy by measuring the energy- and zenith-angle-resolved oscillation probabilities of atmospheric neutrinos traversing the Earth. Additionally, the detector observes a large amount of atmospheric muons, which can be used to study extensive air showers generated by cosmic ray particles.

This work describes a deep-learning based reconstruction of atmospheric muons using graph convolutional networks. They are used to reconstruct the zenith angle, the muon multiplicity and the diameter of atmospheric muon bundles. Simulations and measured data from an early four line stage of the detector are used to evaluate the performance. Furthermore, the reconstructions are compared to the ones of classical approaches, and use cases for the indirect study of cosmic ray particles are shown.

$37^{\text {th }}$ International Cosmic Ray Conference (ICRC 2021)

July 12th - 23rd, 2021

Online - Berlin, Germany

\footnotetext{
${ }^{*}$ Presenter
} 


\section{Atmospheric muons in KM3NeT/ORCA}

ORCA is part of the neutrino detector network KM3NeT [1], which consists of two water Cherenkov detectors currently under construction in the Mediterranean sea, making use of Cherenkov light emitted by fast charged particles in sea water. This light is measured by photomultipliers (PMTs) which are housed in glass spheres (DOMs). Each DOM houses 31 PMTs, which provide additional directional information of the measured photons. In total, ORCA will consist of 7 mega-tons of sea water, instrumented with a 3D-array of 2070 DOMs arranged on 115 strings. For this analysis, simulations and data from the initial four-line stage of the detector is used.

ORCA's primary goal is to study neutrino properties by detecting interactions of atmospheric neutrinos in the $\mathrm{GeV}$ energy range. However, the overwhelming majority of recorded events are actually atmospheric muons produced in cosmic ray induced air showers, which usually pose as a background for neutrino measurements. But they provide a wide range of applications as well, like a validation of the detector performance or measuring the cosmic ray composition. Due to the high rates at which they are detected, the available statistics are much larger than those for neutrinos.

Reconstructing the properties of atmospheric muons can prove challenging. Especially if there are multiple muons crossing the detector at the same time (muon bundles), the signature left behind in the detector can be very complex. Deep Learning methods can drastically simplify the process of analysing these signatures by providing a universal and simple way of reconstructing any observable. Since the data recorded by KM3NeT closely resembles point clouds, Graph Neural networks are a natural choice for the architecture.

\section{Graph neural networks}

For each hit in an event, KM3NeT records a set of coordinates that describe the position and time of where and when the corresponding photon was detected. Among these coordinates are the time, the XYZ-position of the PMT that recorded a hit, as well as the pointing direction of the PMT. In the input to the graph network, these coordinates are the node features [2] of a directional graph, each hit being represented by a single node. The orientation of the PMT is encoded as the three components of a Cartesian unit vector, resulting in seven-dimensional node features.

The edge features of the graph are computed dynamically as part of the network. These dynamic re-computations can play a similar role as the pooling layers in image convolutional networks, since they allow the network to move distant nodes closer together, or vice versa. The architecture of the graph network used in this work resembles the ParticleNet model proposed by Qu et al. [3]. It consists out of three Edge Convolutional blocks, followed by a global pooling layer and two fully connected layers. A custom open-source implementation for this architecture was developed using the tensorflow [4] back end ${ }^{1}$.

\section{Reconstructions}

This section describes the reconstruction of the zenith angle, the multiplicity and the diameter of atmospheric muon bundles, each with its own neural network. A large amount of labelled data is

\footnotetext{
${ }^{1}$ See https://github. com/StefReck/MEdgeConv.
} 
needed to train the networks. For this, a set of 25 million atmospheric muon events was simulated using the software package MUPAGE [5] for the four-line set-up of the ORCA detector. It uses parametric formulas to model the dependence of the flux on e.g. the energy, multiplicity and zenith. All of these events pass at least one of the trigger algorithms described in Ref. [1] and [6]. To combat overfitting, the data is split threefold into 19 million events for training, 1 million events for validating during the training, and 5 million events for generating the plots shown in this work. The training is conducted using the ADAM [7] optimizer and a learning rate of 0.001, which is gradually and exponentially reduced by a factor of 100 over the course of the training. The model takes five days on a GTX 1080ti GPU to fully converge, and requires 2 GB (peak) of VRAM with a batchsize of 64 .

\subsection{Zenith angle}

The goal is to reconstruct the zenith angle of an incident muon or muon bundle. In general, the different muons in a muon bundle have slightly different zenith angles. Since these deviations are usually too small to be resolved, the muons are assumed to be parallel in the MUPAGE simulations, and therefore also in this work. The network reconstructs the incident direction as the components of a three-dimensional unit vector, from which the zenith angle can be computed afterwards. In order to also have access to an estimator for the error of the reconstruction, a probability distribution for each component of the vector is used as the output of the network (see section section 4). The outputs are chosen to be three independent normal distributions $(\mu, \sigma)$, ignoring potential correlations between the components in order to simplify the task.

Figure 1 shows the zenith angle reconstruction performance in comparison to a classical maximum-likelihood based algorithm for single tracks described in [1]. Deep Learning provides a comparable precision on average atmospheric muon events, but shows a substantial improvement for the rarer multi muon events, which make up about a fifth of the dataset. Since the classical method used here was not optimized or intended for atmospheric muon bundles, Deep Learning has an advantage on these events.

In Figure 2, a data-MC comparison of the reconstructed zenith angle of single muon events for both the classical and the deep learning approach is shown. The agreement between the two algorithms is excellent, both on data and simulations. The deviation from one in the data-MC ratio is visible for both approaches, and can be used to tune the simulations in the future.

\subsection{Muon multiplicity}

The number of muons in an event is an important observable for indirectly studying cosmic rays. This deep learning approach is the first reconstruction of the muon multiplicity in KM3NeT. The muon multiplicity is defined here as the number of "reconstructable" atmospheric muons in an event. In order for a muon to qualify as reconstructable, it needs to produce at least 5 hits in the detector. Despite the discrete nature of the multiplicity, the output of the network is set to be a continuous log-normal distribution with a fixed width (sigma) of one. Even though this is a suboptimal posterior, this approach still provides a good estimator for the muon multiplicity (see Figure 3).

The muon multiplicity can be used for the identification of primary particles. Figure 4 shows the result of applying the trained network on 250,000 atmospheric muon events simulated with 


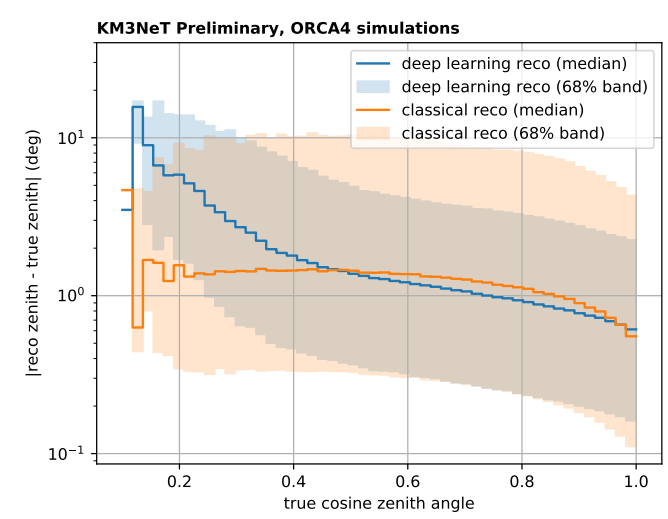

(a) all events

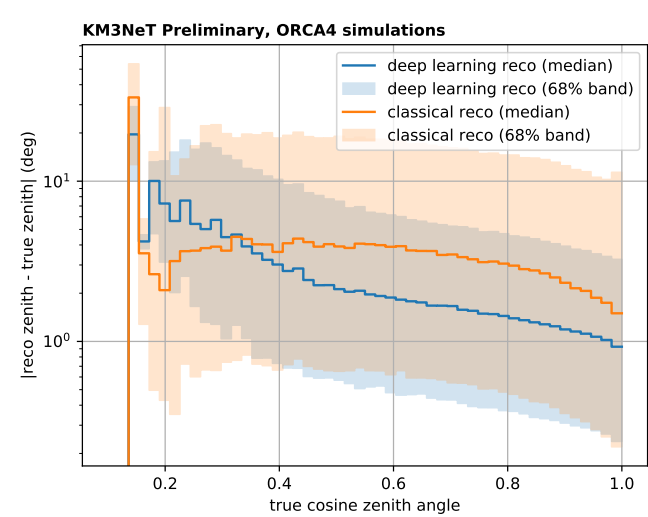

(b) events with two or more muons

Figure 1: Absolute difference between reconstructed and true zenith angle plotted over the true zenith angle for selected atmospheric muons in ORCA4. Shown are the median and the $68 \%$ band for the classical reco (orange) and the deep learning reco (blue). Since it was trained on the expected distribution, the deep learning reconstruction is biased for true cosine zeniths below 0.5, leading to an increase in the error. Most atmospheric muons are not in that region. Deep learning provides a slight improvement in the median for all events (left), which is mostly due to events with two or more muons (right).

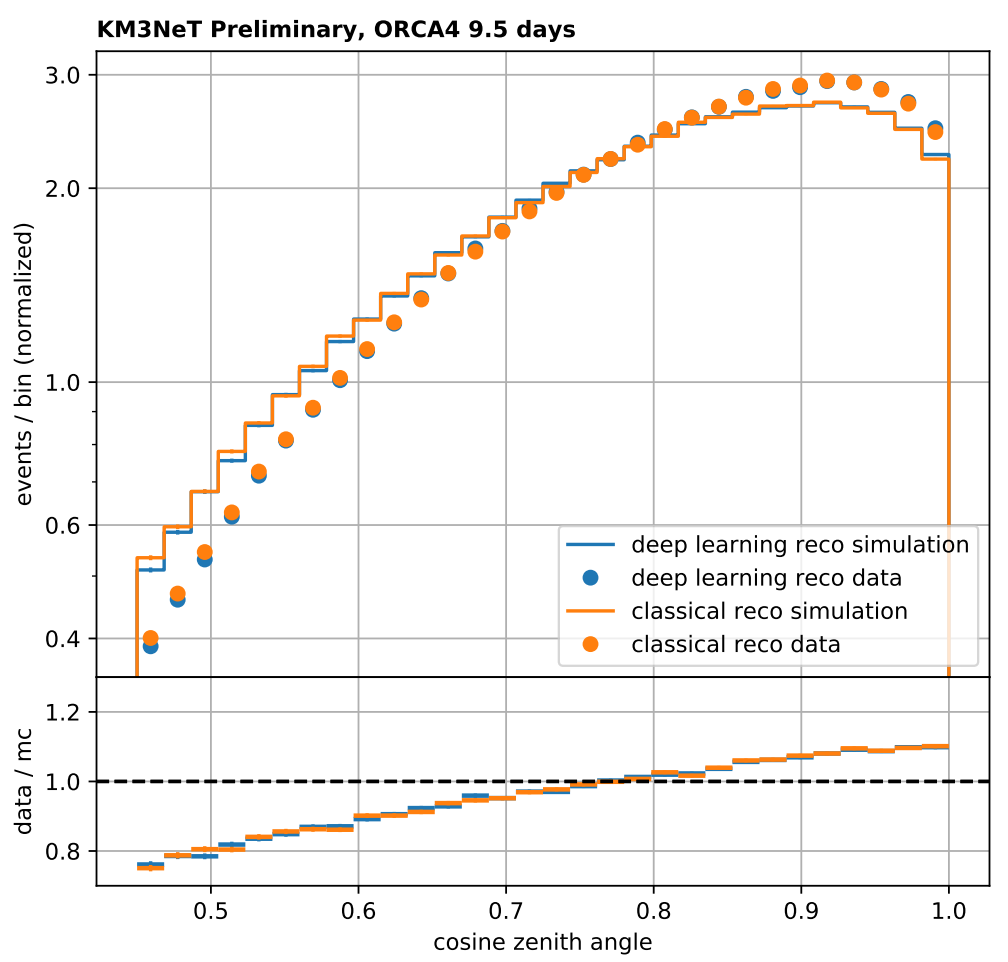

Figure 2: Data-MC comparison of the reconstructed zenith angle for the classical approach (orange) and deep learning (blue). A cut is used on the classical reconstruction quality in order to remove noise and multi-muon events. Each curve is normalized to have an integral of 1, so only the shapes are compared in this plot. 
Muon multiplicity reco for events between 500 and 600 hits

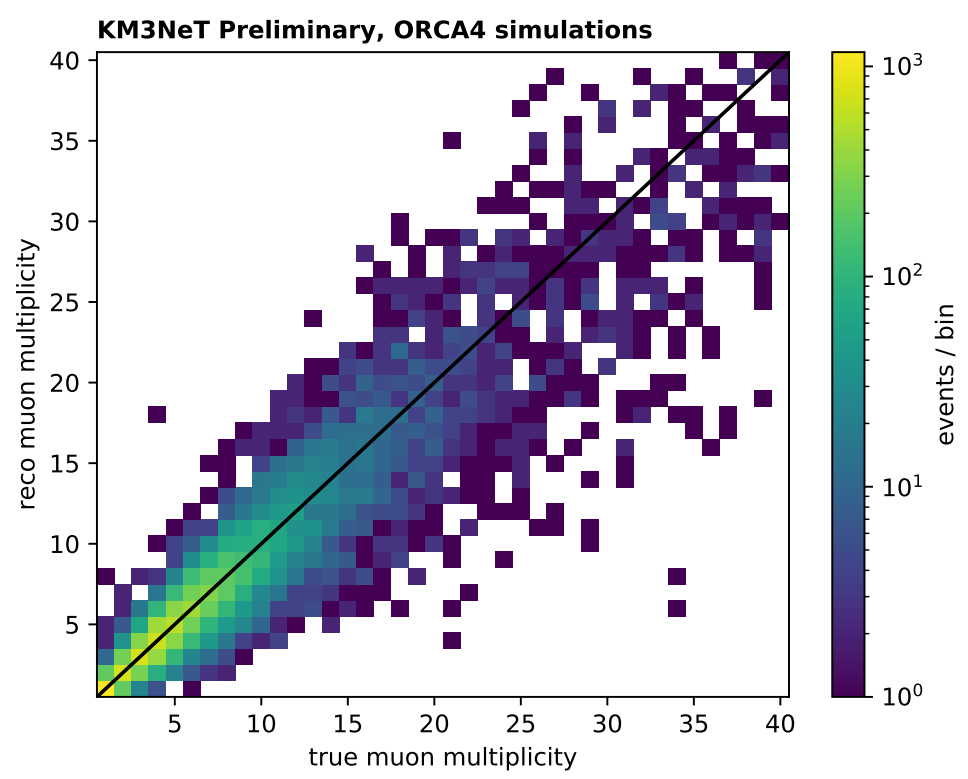

Figure 3: Reconstructed versus true muon multiplicity. The multiplicity is in general correlated to the number of hits, since more muons produce more light on average. The plot only shows events with a comparable number of hits (between 500 and 600). Even in this particularly difficult case, the reconstruction is meaningful.

Corsika [8] SIBYLL 2.3c [9] using the GST-3 [10] spectrum. Despite being in an early stage of construction, the detector already provides a decent separation power between iron and proton induced events.

\subsection{Bundle diameter}

When multiple muons travel through the detector at the same time, a characteristic observable of the bundle is the lateral spread of the muons. Higher energetic primary particles tend to produce bundles with a larger diameter. For the first time in KM3NeT, a reconstruction for the diameter of muon bundles was developed. The muon bundle diameter is here defined as the maximum perpendicular distance between any pair of reconstructable muons in the bundle at the height of the detector.

The output of the network is set to be a log-normal distribution, which is implemented for the sake of simplicity as a normal distribution on the log-transformed bundle diameter. Figure 5 shows the performance of the reconstruction. Since the ORCA4 detector is still in an early stage of construction and therefore quite small, events are often difficult to reconstruct, e.g. because the muons are too far away from the instrumented volume of the detector. These events can be removed with a cut on the reconstructed uncertainty (see section 4). Removing $50 \%$ of the total events with 2 or more muons provides a stable reconstruction performance over a large interval of diameters. 


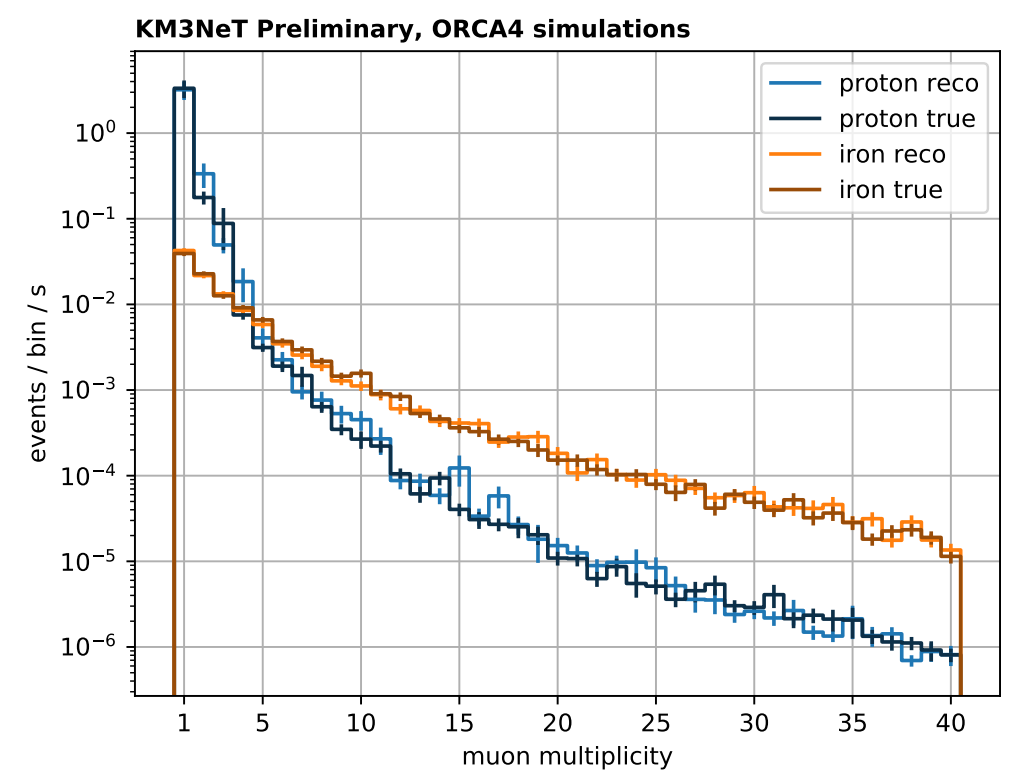

Figure 4: Reconstructed (light) and true (dark) muon multiplicity rates for events generated in proton (blue) and iron (orange) induced atmospheric showers.

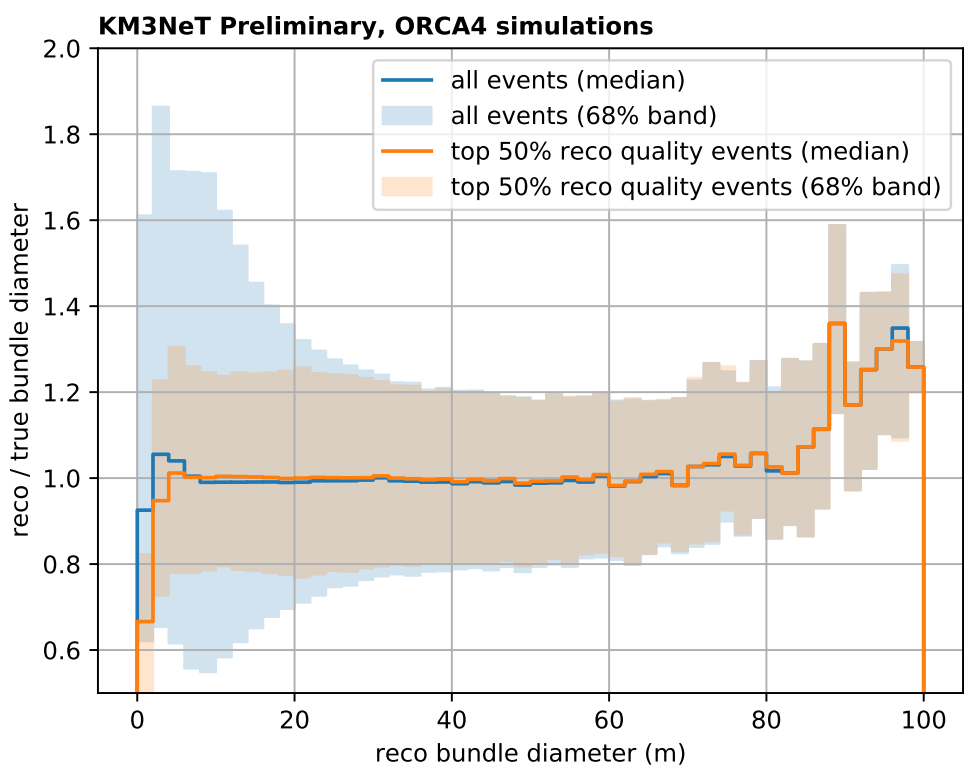

Figure 5: Performance of the bundle diameter reconstruction on events with 2 or more muons. Blue shows all events, orange shows the best $50 \%$ of events according to the reconstructed uncertainty. Events with a high uncertainty tend to get reconstructed at around 10 meters, since this is the maximum of the true distribution. 
Pull distribution for cosine zenith and bundle diameter

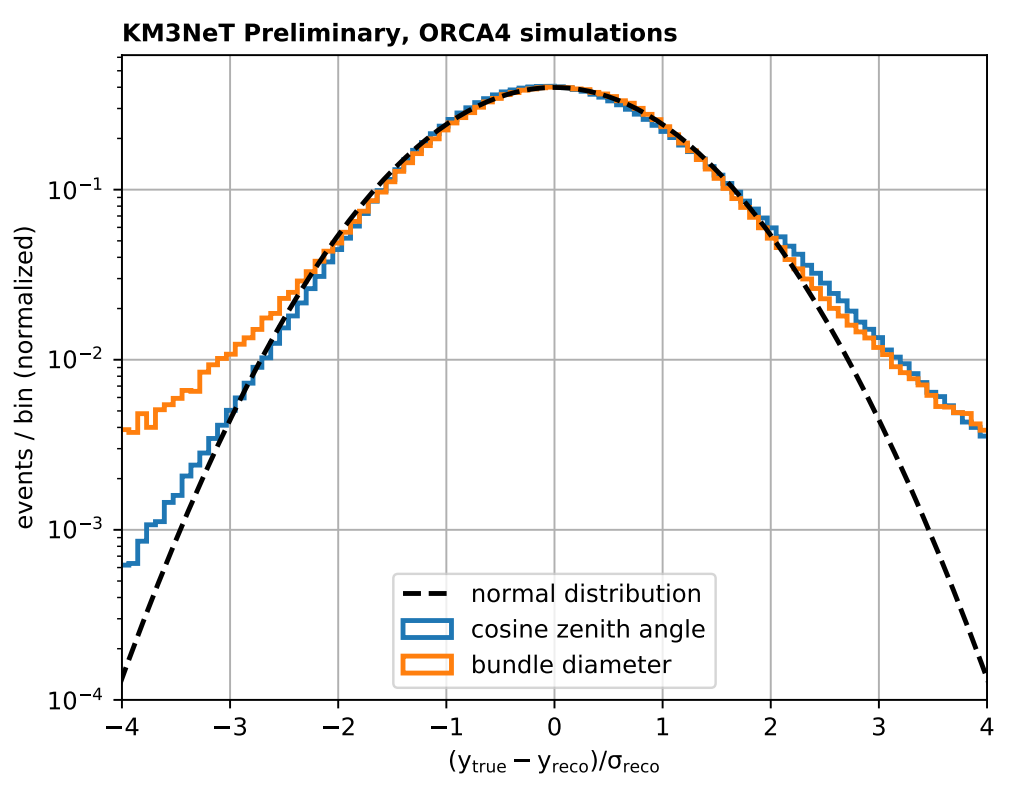

Figure 6: Pull distribution $(\mu-$ true) $/ \sigma$ of the reconstructed cosine zenith angle (blue) and bundle diameter (orange). The dashed black line is the ideal normal pull distribution.

\section{Uncertainty reconstruction}

Deep Learning can be used to provide an estimate of the uncertainty of the reconstruction for each event. For this, the output of the network can be viewed as a probability distribution. For example, the output could be a rational number $\mu$ and a rational positive number $\sigma$, which together make up a normal distribution. The loss to minimize during training can then be set as the negative log-likelihood of the true value given this distribution.

To check the quality of the uncertainty reconstruction, one can take a look at the pull distribution, defined as $(\mu-$ true $) / \sigma$. This is shown in Figure 6 for the cosine zenith angle and bundle diameter reconstructions shown in the previous sections. The distributions agree well with a normal distribution for up to two sigma, indicating a correct uncertainty estimate in that range.

\section{Conclusion}

Graph neural networks have been successfully applied for reconstructing properties of atmospheric muons on four lines of the KM3NeT/ORCA detector. They provide a zenith reconstruction consistent to an established classical algorithm on both data and simulations for single-muon events. Furthermore, they show improvements for multi-muon events, and allow for reconstructing new observables like the muon multiplicity and the bundle diameter. This can be used for validating the detector and indirectly studying the properties of cosmic ray particles. 


\section{References}

[1] S. Adrián-Martínez et al. (KM3NeT Collaboration), Letter of intent for KM3NeT 2.0, Journal of Physics G: Nuclear and Particle Physics, 43 (8), 084001, 2016 [1601.07459v2].

[2] J. Zhou, G. Cui, S. Hu, Z. Zhang, C. Yang, Z. Liu et al., Graph neural networks: A review of methods and applications, 1812.08434.

[3] H. Qu and L. Gouskos, ParticleNet: Jet tagging via particle clouds, Phys. Rev. D 101, 056019 (2020) [1902.08570v3].

[4] M. Abadi et al., TensorFlow: Large-scale machine learning on heterogeneous distributed systems, $1603.04467 \mathrm{v} 2$.

[5] G. Carminati, A. Margiotta and M. Spurio, Atmospheric muons from parametric formulas: a fast generator for neutrino telescopes (MUPAGE), Comput.Phys. Commun.179:915-923,2008 [0802.0562v2].

[6] S. Aiello et al. (KM3NeT Collaboration), Determining the neutrino mass ordering and oscillation parameters with KM3NeT/ORCA, 2103.09885.

[7] D.P. Kingma and J. Ba, Adam: A method for stochastic optimization, 1412.6980v9.

[8] D. Heck, J. Knapp, J. Capdevielle, G. Schatz and T. Thouw, CORSIKA: A Monte Carlo code to simulate extensive air showers, FZKA-6019 (1998) .

[9] F. Riehn, H.P. Dembinski, R. Engel, A. Fedynitch, T.K. Gaisser and T. Stanev, The hadronic interaction model SIBYLL 2.3c and feynman scaling, PoS ICRC2017 (2017) 301 [1709.07227v1].

[10] T.K. Gaisser, T. Stanev and S. Tilav, Cosmic ray energy spectrum from measurements of air showers, Frontiers of Physics (2013) [1303.3565v1]. 


\section{Full Author List: KM3NeT Collaboration}

M. Ageron ${ }^{1}$, S. Aiello ${ }^{2}$, A. Albert ${ }^{3,55}$, M. Alshamsi ${ }^{4}$, S. Alves Garre ${ }^{5}$,Z. Aly ${ }^{1}$, A. Ambrosone ${ }^{6,7}$, F. Ameli ${ }^{8}$, M. Andre ${ }^{9}$, G. Androulakis ${ }^{10}$, M. Anghinolfi ${ }^{11}$, M. Anguita ${ }^{12}$, G. Anton ${ }^{13}$, M. Ardid ${ }^{14}$, S. $\operatorname{Ardid}^{14}$, W. Assal ${ }^{1}$, J. Aublin ${ }^{4}$, C. Bagatelas ${ }^{10}$, B. Baret ${ }^{4}$, S. Basegmez du Pree ${ }^{15}$, M. Bendahman ${ }^{4,16}$, F. Benfenati ${ }^{17,18}$, E. Berbee ${ }^{15}$, A. M. van den Berg ${ }^{19}$, V. Bertin ${ }^{1}$, S. Beurthey ${ }^{1}$, V. van Beveren ${ }^{15}$, S. Biagi ${ }^{20}$, M. Billault ${ }^{1}$, M. Bissinger ${ }^{13}$, M. Boettcher ${ }^{21}$, M. Bou Cabo ${ }^{22}$, J. Boumaaza ${ }^{6}$, M. Bouta ${ }^{23}$, C. Boutonnet ${ }^{4}$, G. Bouvet ${ }^{24}$, M. Bouwhuis ${ }^{15}$, C. Bozza ${ }^{25}$, H.Brânzaş ${ }^{26}$, R. Bruijn ${ }^{15,27}$, J. Brunner ${ }^{1}$, R. Bruno ${ }^{2}$, E. Buis ${ }^{28}$, R. Buompane ${ }^{6,29}$, J. Busto ${ }^{1}$, B. Caiffi ${ }^{11}$, L. Caillat ${ }^{1}$, D. Calvo ${ }^{5}$, S. Campion ${ }^{30,8}$, A. Capone ${ }^{30,8}$, H. Carduner ${ }^{24}$, V. Carretero ${ }^{5}$, P. Castaldi ${ }^{17,31}$, S. Celli ${ }^{30,8}$, R. Cereseto ${ }^{11}$, M. Chabab ${ }^{32}$, C. Champion ${ }^{4}$, N. $\mathrm{Chau}^{4}$, A. Chen ${ }^{33}$, S. Cherubinini ${ }^{20,34}$, V. Chiarella ${ }^{35}$, T. Chiarusi ${ }^{17}$, M. Circella ${ }^{36}$, R. Cocimano ${ }^{20}$, J. A. B. Coelho ${ }^{4}$, A. Coleiro ${ }^{4}$, M. Colomer Molla ${ }^{4,5}$, S. Colonges ${ }^{4}$, R. Coniglione ${ }^{20}$, A. Cosquer ${ }^{1}$, P. Coyle ${ }^{1}$, M. Cresta ${ }^{11}$, A. Creusot ${ }^{4}$, A. $\mathrm{Cruz}^{37}$, G. Cuttone ${ }^{20}$, A. D’Amico ${ }^{15}$, R. Dallier ${ }^{24}$, B. De Martino ${ }^{1}$, M. De Palma ${ }^{36,38}$, I. Di Palma ${ }^{30,8}$, A. F. Díaz ${ }^{12}$, D. Diego$\operatorname{Tortosa}^{14}$, C. Distefano ${ }^{20}$, A. Domi ${ }^{15,27}$, C. Donzaud ${ }^{4}$, D. Dornic ${ }^{1}$, M. Dörr ${ }^{39}$, D. Drouhin ${ }^{3,55}$, T. Eberl ${ }^{13}$, A. Eddyamoui ${ }^{16}$, T. van Eeden ${ }^{15}$, D. van Eijk ${ }^{15}$, I. El Bojaddaini ${ }^{23}$, H. Eljarrari ${ }^{16}$, D. Elsaesser ${ }^{39}$, A. Enzenhöfer ${ }^{1}$, V. Espinosa ${ }^{14}$, P. Fermani ${ }^{30,8}$, G. Ferrara ${ }^{20,34}$, M. D. Filipović ${ }^{40}$, F. Filippini ${ }^{17,18}$, J. Fransen ${ }^{15}$, L. A. Fusco ${ }^{1}$, D. Gajanana ${ }^{15}$, T. Gal ${ }^{13}$, J. García Méndez ${ }^{14}$, A. Garcia Soto $^{5}$, E. Garçon ${ }^{1}$, F. Garufi ${ }^{6,7}$, C. Gatius ${ }^{15}$, N. Geißelbrecht ${ }^{13}$, L. Gialanella ${ }^{6,29}$, E. Giorgio ${ }^{20}$, S. R. Gozzini ${ }^{5}$, R. Gracia ${ }^{15}$, K. Graf $^{13}$, G. Grella ${ }^{41}$, D. Guderian ${ }^{56}$, C. Guidi ${ }^{11,42}$, B. Guillon ${ }^{43}$, M. Gutiérrez $z^{44}$, J. Haefner ${ }^{13}$, S. Hallmann ${ }^{13}$, H. Hamdaoui ${ }^{16}$, H. van Haren ${ }^{45}$, A. Heijboer ${ }^{15}$, A. Hekalo ${ }^{39}$, L. Hennig ${ }^{13}$, S. Henry ${ }^{1}$, J. J. Hernández-Rey ${ }^{5}$, J. Hofestädt ${ }^{13}$, F. Huang ${ }^{1}$, W. Idrissi Ibnsalih ${ }^{6,29}$, A. Ilioni ${ }^{4}$, G. Illuminati ${ }^{17,18,4}$, C. W. James ${ }^{37}$, D. Janezashvili ${ }^{46}$, P. Jansweijer ${ }^{15}$, M. de Jong ${ }^{15,47}$, P. de Jong ${ }^{15,27}$, B. J. Jung ${ }^{15}$, M. Kadler ${ }^{39}$, P. Kalaczyński ${ }^{48}$, O. Kalekin ${ }^{13}$, U. F. Katz ${ }^{13}$, F. Kayzel ${ }^{15}$, P. Keller ${ }^{1}$, N. R. Khan Chowdhury ${ }^{5}$, G. Kistauri ${ }^{46}$, F. van der Knaap ${ }^{28}$, P. Kooijman ${ }^{27,57}$, A. Kouchner ${ }^{4,49}$, M. Kreter ${ }^{21}$, V. Kulikovskiy ${ }^{11}$, M. Labalme ${ }^{43}$, P. Lagier ${ }^{1}$, R. Lahmann ${ }^{13}$, P. Lamare ${ }^{1}$, M. Lamoureux $^{24}$, G. Larosa ${ }^{20}$, C. Lastoria ${ }^{1}$, J. Laurence ${ }^{1}$, A. Lazo $^{5}$, R. Le Breton ${ }^{4}$, E. Le Guirriec ${ }^{1}$, S. Le Stum ${ }^{1}$, G. Lehaut ${ }^{43}$, O. Leonardi ${ }^{20}$, F. Leone ${ }^{20,34}$, E. Leonora ${ }^{2}$, C. Lerouvillois ${ }^{1}$, J. Lesrel ${ }^{4}$, N. Lessing ${ }^{13}$, G. Levi ${ }^{17,18}$, M. Lincetto ${ }^{1}$, M. Lindsey Clark ${ }^{4}$, T. Lipreau ${ }^{24}$, C. LLorens Alvarez ${ }^{14}$, A. Lonardo ${ }^{8}$, F. Longhitano ${ }^{2}$, D. Lopez-Coto ${ }^{44}$, N. Lumb ${ }^{1}$, L. Maderer ${ }^{4}$, J. Majumdar ${ }^{15}$, J. Mańczak ${ }^{5}$, A. Margiotta ${ }^{17,18}$, A. Marinelli ${ }^{6}$, A. Marini ${ }^{1}$, C. Markou $^{10}$, L. Martin ${ }^{24}$, J. A. Martínez-Mora ${ }^{14}$, A. Martini ${ }^{35}$, F. Marzaioli ${ }^{6,29}$,

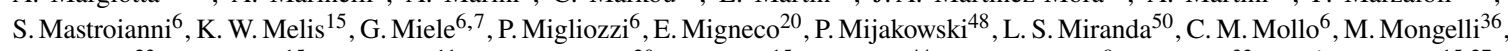
A. Moussa ${ }^{23}$, R. Muller ${ }^{15}$, P. Musico ${ }^{11}$, M. Musumeci ${ }^{20}$, L. Nauta ${ }^{15}$, S. Navas ${ }^{44}$, C. A. Nicolau ${ }^{8}$, B. Nkosi ${ }^{33}$, B. Ó Fearraigh ${ }^{15,27}$, M. O'Sullivan ${ }^{37}$, A. Orlando ${ }^{20}$, G. Ottonello ${ }^{11}$, S. Ottonello ${ }^{11}$, J. Palacios González ${ }^{5}$, G. Papalashviliit ${ }^{46}$, R. Papaleo ${ }^{20}$, C. Pastore ${ }^{36}$, A. M. Păun ${ }^{26}$, G. E. Păvălaş ${ }^{26}$, G. Pellegrinini ${ }^{17}$, C. Pellegrino ${ }^{18,58}$, M. Perrin-Terrini ${ }^{1}$, V. Pestel ${ }^{15}$, P. Piattelli ${ }^{20}$, C. Pieterse ${ }^{5}$, O. Pisanti ${ }^{6,7}$, C. Poirè ${ }^{14}$, V. Popa $^{26}$, T. Pradier $^{3}$, F. Pratolongo ${ }^{11}$, I. Probst ${ }^{13}$, G. Pühlhofer ${ }^{51}$, S. Pulvirenti ${ }^{20}$, G. Quéméner ${ }^{43}$, N. Randazzo ${ }^{2}$, A. Rapicavoli ${ }^{34}$, S. Razzaque $^{50}$, D. Real ${ }^{5}$, S. Reck ${ }^{13}$, G. Riccobene ${ }^{20}$, L. Rigalleau ${ }^{24}$, A. Romanov ${ }^{11,42}$, A. Rovelli ${ }^{20}$, J. Royon ${ }^{1}$, F. Salesa Greus ${ }^{5}$, D. F. E. Samtleben ${ }^{15,47}$, A. Sánchez Losa ${ }^{36,5}$, M. Sanguineti ${ }^{11,42}$, A. Santangelo ${ }^{51}$, D. Santonocito ${ }^{20}$, P. Sapienza ${ }^{20}$, J. Schmelling ${ }^{15}$, J. Schnabel ${ }^{13}$, M. F. Schneider ${ }^{13}$, J. Schumann ${ }^{13}$, H. M. Schutte ${ }^{21}$, J. Seneca ${ }^{15}$, I. Sgura ${ }^{36}$, R. Shanidze ${ }^{46}$, A. Sharma ${ }^{52}$, A. Sinopoulou ${ }^{10}$, B. Spisso ${ }^{41,6}$, M. Spurio ${ }^{17,18}$, D. Stavropoulos ${ }^{10}$, J. Steijger ${ }^{15}$, S. M. Stellacci ${ }^{41,6}$, M. Taiuti ${ }^{11,42}$, F. Tatone ${ }^{36}$, Y. Tayalati ${ }^{16}$, E. Tenllado ${ }^{44}$, D. Tézier ${ }^{1}$, T. Thakore ${ }^{5}$, S. Theraube ${ }^{1}$, H. Thiersen $^{21}$, P. Timmer $^{15}$, S. Tingay ${ }^{37}$, S. Tsagkli $^{10}$, V. Tsourapis ${ }^{10}$, E. Tzamariudaki ${ }^{10}$, D. Tzanetatos ${ }^{10}$, C. Valieri ${ }^{17}$, V. Van Elewyck ${ }^{4,49}$, G. Vasileiadis ${ }^{53}$, F. Versari ${ }^{17,18}$, S. Viola $^{20}$, D. Vivolo ${ }^{6,29}$, G. de Wasseige ${ }^{4}$, J. Wilms $^{54}$, R. Wojaczyński ${ }^{48}$, E. de Wolf ${ }^{15,27}$, T. Yousfi ${ }^{23}$, S. Zavatarelli ${ }^{11}$, A. Zegarelli ${ }^{30,8}$, D. Zito ${ }^{20}$, J. D. Zornoza ${ }^{5}$, J. Zúñiga ${ }^{5}$, N. Zywucka ${ }^{21}$.

${ }^{1}$ Aix Marseille Univ, CNRS/IN2P3, CPPM, Marseille, France.

${ }^{2}$ INFN, Sezione di Catania, Via Santa Sofia 64, Catania, 95123 Italy.

${ }^{3}$ Université de Strasbourg, CNRS, IPHC UMR 7178, F-67000 Strasbourg, France.

${ }^{4}$ Université de Paris, CNRS, Astroparticule et Cosmologie, F-75013 Paris, France.

${ }^{5}$ IFIC - Instituto de Física Corpuscular (CSIC - Universitat de València), c/Catedrático José Beltrán, 2, 46980 Paterna, Valencia, Spain. ${ }^{6}$ INFN, Sezione di Napoli, Complesso Universitario di Monte S. Angelo, Via Cintia ed. G, Napoli, 80126 Italy.

${ }^{7}$ Università di Napoli “Federico II”, Dip. Scienze Fisiche "E. Pancini”, Complesso Universitario di Monte S. Angelo, Via Cintia ed. G, Napoli, 80126 Italy.

${ }^{8}$ INFN, Sezione di Roma, Piazzale Aldo Moro 2, Roma, 00185 Italy.

${ }^{9}$ Universitat Politècnica de Catalunya, Laboratori d'Aplicacions Bioacústiques, Centre Tecnològic de Vilanova i la Geltrú, Avda. Rambla Exposició, s/n, Vilanova i la Geltrú, 08800 Spain.

${ }^{10}$ NCSR Demokritos, Institute of Nuclear and Particle Physics, Ag. Paraskevi Attikis, Athens, 15310 Greece.

${ }^{11}$ INFN, Sezione di Genova, Via Dodecaneso 33, Genova, 16146 Italy.

${ }^{12}$ University of Granada, Dept. of Computer Architecture and Technology/CITIC, 18071 Granada, Spain.

${ }^{13}$ Friedrich-Alexander-Universität Erlangen-Nürnberg, Erlangen Centre for Astroparticle Physics, Erwin-Rommel-Straße 1, 91058 Erlangen, Germany.

${ }^{14}$ Universitat Politècnica de València, Instituto de Investigación para la Gestión Integrada de las Zonas Costeras, C/ Paranimf, 1, Gandia, 46730 Spain.

${ }^{15}$ Nikhef, National Institute for Subatomic Physics, PO Box 41882, Amsterdam, 1009 DB Netherlands.

${ }^{16}$ University Mohammed V in Rabat, Faculty of Sciences, 4 av. Ibn Battouta, B.P. 1014, R.P. 10000 Rabat, Morocco.

${ }^{17}$ INFN, Sezione di Bologna, v.le C. Berti-Pichat, 6/2, Bologna, 40127 Italy.

${ }^{2}$ also at Dipartimento di Fisica, INFN Sezione di Padova and Università di Padova, I-35131, Padova, Italy 
${ }^{18}$ Università di Bologna, Dipartimento di Fisica e Astronomia, v.le C. Berti-Pichat, 6/2, Bologna, 40127 Italy.

${ }^{19}$ KVI-CART University of Groningen, Groningen, the Netherlands.

${ }^{20}$ INFN, Laboratori Nazionali del Sud, Via S. Sofia 62, Catania, 95123 Italy.

${ }^{21}$ North-West University, Centre for Space Research, Private Bag X6001, Potchefstroom, 2520 South Africa.

${ }^{22}$ Instituto Español de Oceanografía, Unidad Mixta IEO-UPV, C/ Paranimf, 1, Gandia, 46730 Spain.

${ }^{23}$ University Mohammed I, Faculty of Sciences, BV Mohammed VI, B.P. 717, R.P. 60000 Oujda, Morocco.

${ }^{24}$ Subatech, IMT Atlantique, IN2P3-CNRS, Université de Nantes, 4 rue Alfred Kastler - La Chantrerie, Nantes, BP 2072244307 France.

${ }^{25}$ Università di Salerno e INFN Gruppo Collegato di Salerno, Dipartimento di Matematica, Via Giovanni Paolo II 132, Fisciano, 84084 Italy.

${ }^{26}$ ISS, Atomistilor 409, Măgurele, RO-077125 Romania

${ }^{27}$ University of Amsterdam, Institute of Physics/IHEF, PO Box 94216, Amsterdam, 1090 GE Netherlands.

${ }^{28}$ TNO, Technical Sciences, PO Box 155, Delft, 2600 AD Netherlands.

${ }^{29}$ Università degli Studi della Campania "Luigi Vanvitelli", Dipartimento di Matematica e Fisica, viale Lincoln 5, Caserta, 81100 Italy.

${ }^{30}$ Università La Sapienza, Dipartimento di Fisica, Piazzale Aldo Moro 2, Roma, 00185 Italy.

${ }^{31}$ Università di Bologna, Dipartimento di Ingegneria dell'Energia Elettrica e dell'Informazione "Guglielmo Marconi", Via dell'Università 50, Cesena, 47521 Italia.

${ }^{32}$ Cadi Ayyad University, Physics Department, Faculty of Science Semlalia, Av. My Abdellah, P.O.B. 2390, Marrakech, 40000 Morocco.

${ }^{33}$ University of the Witwatersrand, School of Physics, Private Bag 3, Johannesburg, Wits 2050 South Africa.

${ }^{34}$ Università di Catania, Dipartimento di Fisica e Astronomia "Ettore Majorana", Via Santa Sofia 64, Catania, 95123 Italy.

${ }^{35}$ INFN, LNF, Via Enrico Fermi, 40, Frascati, 00044 Italy.

${ }^{36}$ INFN, Sezione di Bari, via Orabona, 4, Bari, 70125 Italy.

${ }^{37}$ International Centre for Radio Astronomy Research, Curtin University, Bentley, WA 6102, Australia.

${ }^{38}$ University of Bari, Via Amendola 173, Bari, 70126 Italy.

${ }^{39}$ University Würzburg, Emil-Fischer-Straße 31, Würzburg, 97074 Germany.

${ }^{40}$ Western Sydney University, School of Computing, Engineering and Mathematics, Locked Bag 1797, Penrith, NSW 2751 Australia.

${ }^{41}$ Università di Salerno e INFN Gruppo Collegato di Salerno, Dipartimento di Fisica, Via Giovanni Paolo II 132, Fisciano, 84084 Italy.

${ }^{42}$ Università di Genova, Via Dodecaneso 33, Genova, 16146 Italy.

${ }^{43}$ Normandie Univ, ENSICAEN, UNICAEN, CNRS/IN2P3, LPC Caen, LPCCAEN, 6 boulevard Maréchal Juin, Caen, 14050 France.

${ }^{44}$ University of Granada, Dpto. de Física Teórica y del Cosmos \& C.A.F.P.E., 18071 Granada, Spain.

${ }^{45} \mathrm{NIOZ}$ (Royal Netherlands Institute for Sea Research), PO Box 59, Den Burg, Texel, 1790 AB, the Netherlands.

${ }^{46}$ Tbilisi State University, Department of Physics, 3, Chavchavadze Ave., Tbilisi, 0179 Georgia.

${ }^{47}$ Leiden University, Leiden Institute of Physics, PO Box 9504, Leiden, 2300 RA Netherlands.

${ }^{48}$ National Centre for Nuclear Research, 02-093 Warsaw, Poland.

${ }^{49}$ Institut Universitaire de France, 1 rue Descartes, Paris, 75005 France.

${ }^{50}$ University of Johannesburg, Department Physics, PO Box 524, Auckland Park, 2006 South Africa.

${ }^{51}$ Eberhard Karls Universität Tübingen, Institut für Astronomie und Astrophysik, Sand 1, Tübingen, 72076 Germany.

${ }^{52}$ Università di Pisa, Dipartimento di Fisica, Largo Bruno Pontecorvo 3, Pisa, 56127 Italy.

${ }^{53}$ Laboratoire Univers et Particules de Montpellier, Place Eugène Bataillon - CC 72, Montpellier Cédex 05, 34095 France.

${ }^{54}$ Friedrich-Alexander-Universität Erlangen-Nürnberg, Remeis Sternwarte, Sternwartstraße 7, 96049 Bamberg, Germany.

${ }^{55}$ Université de Haute Alsace, 68100 Mulhouse Cedex, France.

${ }^{56}$ University of Münster, Institut für Kernphysik, Wilhelm-Klemm-Str. 9, Münster, 48149 Germany.

${ }^{57}$ Utrecht University, Department of Physics and Astronomy, PO Box 80000, Utrecht, 3508 TA Netherlands.

${ }^{58}$ INFN, CNAF, v.le C. Berti-Pichat, 6/2, Bologna, 40127 Italy. 